Pensamiento Crítico Vol.17. N¹, pp. 47-65

\title{
La valorización de una empresa
}

\author{
Dr. Raimundo Renaun Pacheco Mexzon
}

\section{RESUMEN}

La empresa como un negocio en marcha generador de fondos. En consecuencia, cuando se quiere determinar su valor en el mercado debe tenerse en cuenta un periodo histórico (pasado), el presente y un periodo futuro. Dentro del periodo futuro debe considerarse un periodo explícito y un periodo continuo. El periodo explícito futuro debe considerar como máximo 5 años y el periodo continuo futuro a partir del año 6 hasta el fin de la vida útil de la empresa. En este contexto trataremos de calcular el valor de la empresa utilizando el método del flujo de caja descontado.

La importancia que ha tomado la adquisición de empresas obliga a utilizar esta técnica de valorización que nos permite calcular el valor de la empresa para que se pueda intentar tener un precio justo.

De allí que nuestra inquietud es plantear una metodología para calcular el valor de la empresa partiendo del "Flujo de caja libre descontado".

Palabras claves: Flujo de caja libre descontado, valor de la empresa.

\section{ABSTRACT}

The company as a going concern generated funds. Therefore when you want to determine its market value should be considered a historical period (the past), present and future periods. In the future period should be considered an explicit 


\section{Pensamiento Crítico Vol.17. No I}

period and a sustained period. The explicit term future must consider more than 5 years and the future continuous period from year 6 to the end of the life of the company. In this context we try to calculate the value of the company using the method of discounted cash flow.

The importance that has taken the acquisitions necessary to use this valuation technique that allows us to calculate the value of the company so you can try for a fair price.

Hence, our concern is to propose a methodology for calculating the enterprise value based on the "discounted free cash flow."

Keywords: free cash flow discounted, value of the company.

\section{Introducción}

El presente trabajo se desarrolló luego de plantearnos las siguientes preguntas:

1. ¿La compra y venta de empresas se pueden realizar calculando el valor de la empresa en el mercado como un negocio en marcha?

2. ¿El flujo de caja libre descontado puede ser útil para determinar el valor de la empresa en el mercado?

Para responderlas se desarrolló el marco teórico que incluye la conceptualización del valor de la empresa a partir del flujo de caja libre descontado, planteándose un modelo sencillo de valorización.

Luego, se plantea la aplicación del modelo desarrollado en el marco teórico tomando información real de una empresa peruana que cotiza en nuestra Bolsa de Valores de Lima. Esta información que aparece en sus estados financieros auditados, permitió hacer las proyecciones respectivas y llegar a la determinación del valor de la empresa.

Se concluye que el flujo de caja libre proyectado descontado es una buena alternativa para calcular el valor de la empresa considerando en las proyecciones un periodo explícito de 5 años y un periodo continuo a partir del año 6 hasta la vida útil de la empresa. 


\section{Raimundo Renaun Pacheco Mexzon}

Se recomienda que esta metodología se utilice para calcular el valor de la empresa si se desea comprarla o venderla.

\section{Marco teórico}

Para calcular el valor de la empresa se presentan una serie de métodos que consideran en términos generales que la empresa tiene una estructura financiera determinada. Es decir, el total de activos de la empresa está financiado con pasivos o deudas y patrimonio o capital propio.

Por lo tanto, partiendo de la teoría del valor de la empresa en el mercado se puede maximizar este valor teniendo una estructura adecuada de capital.

La representación matemática para calcular el valor de la empresa en el mercado es:

$$
\text { V. E. = VAPE + VAPC }
$$

Donde:

V. E. = Valor de la empresa en el mercado

$\mathrm{VAPE}=$ Valor actual del periodo explícito

VAPC $=$ Valor actual del periodo continuo

La estructura de capital o estructura financiera de la empresa es:

Donde:

$$
\text { V.E. }=\text { ACTIVOS }=\text { DEUDA }+ \text { PATRIMONIO }
$$

V. E. = Valor de la empresa en el mercado

ACTIVOS $=$ DEUDA + PATRIMONIO 


\section{Pensamiento Crítico Vol.17. No I}

Contando con lo anteriormente descrito se puede plantear que el máximo valor de la empresa en el mercado será:

$$
\text { Máx. V. E. = k*VE (PATRIMONIO) + } \mathrm{w} * \mathrm{VE}(\text { DEUDA) }
$$

Donde:

Max. V. E. = Máximo valor de la empresa en el mercado

"k" y " $\mathrm{w}$ " son los porcentajes del valor de la empresa que definen el PATRIMONIO y la DEUDA necesarios para determinar la estructura adecuada de capital. Ambos suman "uno" porque representan el 100\%.

Matemáticamente tenemos: $\mathrm{k}+\mathrm{w}=1$ de donde $\mathrm{w}=1-\mathrm{k}$

Luego, el máximo valor de mercado de la empresa se da con la siguiente estructura de capital:

$$
\mathrm{k} * \text { VE como PATRIMONIO + (1-k)*VE como DEUDA }
$$

\section{Objetivo}

El objetivo general del presente trabajo es demostrar que se puede calcular el valor de la empresa en el mercado utilizando la técnica del valor presente neto del "Flujo de caja libre descontado" utilizando un periodo explícito de 5 años y un periodo continuo a partir del año 6 .

\section{Flujo de caja libre (free cash flow)}

\section{Concepto}

El flujo de caja libre (Free Cash Flow) es el flujo de fondos operativo de la empresa que se determina a partir de la utilidad operativa después de impuesto agregándole la depreciación y restándole la inversión en activos fijos y en capital de trabajo. 


\section{Raimundo Renaun Pacheco Mexzon}

Algunos le llaman a la utilidad operativa después de impuestos, "utilidad antes de intereses después de impuestos".

En consecuencia, el flujo de caja libre no incluye gastos financieros ni amortización de deudas.

\section{Un flujo de caja libre}

Si quisiéramos conocer la presentación de un flujo de caja libre veamos el siguiente cuadro:

\section{Flujo de caja libre}

\begin{tabular}{|l|c|c|}
\hline \multicolumn{1}{|c|}{ AÑOS } & $\mathbf{1}$ & $\mathbf{2}$ \\
\hline Ingresos por ventas & 1000 & 1200 \\
\hline Menos costo de ventas & 600 & 750 \\
\hline Utilidad Bruta & 400 & 450 \\
\hline Menos gastos operativos & 180 & 200 \\
\hline $\begin{array}{l}\text { Utilidad operativa } \\
\text { (Utilidad antes de intereses e impuestos) }\end{array}$ & $\mathbf{2 2 0}$ & $\mathbf{2 5 0}$ \\
\hline Impuesto a la renta (30\%) & 66 & 75 \\
\hline Utilidad antes de intereses después de impuestos & $\mathbf{1 5 4}$ & $\mathbf{1 7 5}$ \\
\hline (+) Depreciación & 50 & 60 \\
\hline (-) Inversiones en activos fijos & 70 & 80 \\
\hline (-) Incremento del capital de trabajo & 2 & 3 \\
\hline FLUJO DE CAJA LIBRE & $\mathbf{1 3 2}$ & $\mathbf{1 5 2}$ \\
\hline
\end{tabular}

\section{Un caso práctico}

En esta parte del trabajo se escogerá una empresa que actualmente cotiza en la Bolsa de Valores de Lima y se le aplicará la metodología planteada en el marco teórico.

De esta manera se determinará su estructura adecuada de capital que permita maximizar su valor en el mercado. 


\section{Pensamiento Crítico Vol.I7. No I}

\section{Evolución histórica de la empresa}

Enrique Ferreyros Ayulo y un grupo de socios fundaron en 1922 la empresa Enrique Ferreyros y Cia. La cual se dedicó en sus primeros años a la comercialización de productos de consumo masivo, atendiendo al mercado de abarrotes. Veinte años más tarde la empresa experimenta un giro trascendental, cuando toma la decisión de asumir la representación de Caterpillar Tractor Co. en el Perú. A partir de ese momento la compañía empieza a incursionar en nuevos negocios y a redefinir su cartera de clientes, marcando así el futuro desarrollo de toda la organización.

Dos décadas después, otra línea de máquinas y equipos como Massey Ferguson le encomiendan su representación, sumándose a estas más adelante marcas como Ingersoll Rand, Chevrolet, Kenworth y otras.

En 1966, la empresa modifica su antigua razón social por la de Ferreyros S.A., como parte de un proceso de modernización a fin de mostrar la nueva estructura accionaria.

Este cambio fue reforzado por la introducción de una nueva imagen, en la que se introduce el símbolo Magis "Mas", que resume el compromiso de la empresa por ofrecer cada día más valor, más servicios y respaldo total a sus clientes.

De acuerdo con su estatuto, Ferreyros tiene por objetivo la compra y venta de mercaderías y productos nacionales y extranjeros, la importación y exportación de mercaderías y artículos en general, la provisión de servicios y realización de inversiones y comisiones.

Al 31 de diciembre del 2001, el capital social está representado por 161'850,384 acciones comunes de un valor nominal de S/. 1.095 cada una , íntegramente suscritas y pagadas de las cuales $83.20 \%$ pertenecen a accionistas nacionales y $16.80 \%$ a inversionistas extranjeros.

Ferreyros, reconocida como la principal distribuidora de bienes de capital en el Perú, destina sus productos hacia una gama diversa de sectores de la actividad económica como minería, construcción, agricultura, energía, pesca y transporte. Es la única distribuidora de Caterpillar en el Perú desde 1942. 


\section{Raimundo Renaun Pacheco Mexzon}

La compañía mantiene inversiones en varias subsidiarias. Al 31 de diciembre del 2001, su participación en el capital social de las mismas fue como sigue:

Orvisa S.A. 99\%, Heavy Machinery Services Ltd 100\%, Fiansa S.A. 99\%, Depositos Efe S.A. 99\%, Motorindustria S.A. 99.9\%, Domingo Rodas S.A. 27.5\%, Unimaq S.A. 99.9\%, y Matreq Ferreyros S.A. 99.9\%.

\section{Análisis de los resultados históricos}

A continuación se presenta el resumen de los balances para el periodo 1997 - 2001.

\section{Resumen histórico del balance general}

En miles de soles

\begin{tabular}{|l|c|c|c|c|c|}
\hline & $\mathbf{2 0 0 1}$ & $\mathbf{2 0 0 0}$ & $\mathbf{1 9 9 9}$ & $\mathbf{1 9 9 8}$ & $\mathbf{1 9 9 7}$ \\
\hline $\begin{array}{l}\text { ACTIVO } \\
\text { CORRIENTE }\end{array}$ & $\mathbf{4 5 2 , 5 5 4}$ & $\mathbf{5 7 1 , 0 4 8}$ & $\mathbf{5 2 5 , 2 0 7}$ & $\mathbf{5 7 6 , 6 3 0}$ & $\mathbf{4 7 1 , 9 4 1}$ \\
\hline Caja/Bancos & 19,963 & 32,738 & 17,312 & 19,670 & 19,524 \\
\hline $\begin{array}{l}\text { Cuentas por cobrar } \\
\text { comerciales }\end{array}$ & 102,329 & 179,981 & 180,076 & 97,663 & 159,817 \\
\hline $\begin{array}{l}\text { Otras cuentas por } \\
\text { cobrar }\end{array}$ & 34,170 & 78,288 & 90,185 & 151,807 & 75,088 \\
\hline Existencias & 296,092 & 280,041 & 237,634 & 307,490 & 217,512 \\
\hline $\begin{array}{l}\text { ACTIVO NO } \\
\text { CORRIENTE }\end{array}$ & $\mathbf{3 9 9 , 5 9 8}$ & $\mathbf{3 4 9 , 1 6 0}$ & $\mathbf{2 9 8 , 0 9 8}$ & $\mathbf{2 3 5 , 7 9 8}$ & $\mathbf{2 8 0 , 6 5 6}$ \\
\hline Activo fijo neto & 232,178 & 247,629 & 191,383 & 152,480 & 189,374 \\
\hline Otras inversiones & 127,357 & 76,497 & 76,736 & 71,961 & 55,600 \\
\hline Ctas. por Cobrar L. P. & 40,063 & 25,034 & 29,979 & 11,357 & 35,682 \\
\hline TOTAL ACTIVOS & $\mathbf{8 5 2 , 1 5 2}$ & $\mathbf{9 2 0 , 2 0 8}$ & $\mathbf{8 2 3 , 3 0 5}$ & $\mathbf{8 1 2 , 4 2 8}$ & $\mathbf{7 5 2 , 5 9 7}$ \\
\hline
\end{tabular}


Pensamiento Crítico Vol.17. No I

\begin{tabular}{|l|c|c|c|c|c|}
\hline $\begin{array}{l}\text { PASIVO } \\
\text { CORRIENTE }\end{array}$ & $\mathbf{4 9 2 , 7 4 1}$ & $\mathbf{4 3 5 , 3 7 3}$ & $\mathbf{3 6 2 , 1 0 3}$ & $\mathbf{4 6 4 , 5 6 2}$ & $\mathbf{3 3 3 , 9 0 3}$ \\
\hline Préstamos bancarios & 146,871 & 177,070 & 109,532 & 920 & 1,146 \\
\hline $\begin{array}{l}\text { Cuentas por pagar } \\
\text { comerciales }\end{array}$ & 119,419 & 132,793 & 106,234 & 99,563 & 100,020 \\
\hline $\begin{array}{l}\text { Otras cuentas por } \\
\text { pagar }\end{array}$ & 26,645 & 35,835 & 26,522 & 191,822 & 106,359 \\
\hline $\begin{array}{l}\text { Parte corriente deuda } \\
\text { de L. P. }\end{array}$ & 199,806 & 89,675 & 119,815 & 172,257 & 126,378 \\
\hline $\begin{array}{l}\text { PASIVO NO } \\
\text { CORRIENTE }\end{array}$ & $\mathbf{1 3 4 , 3 0 4}$ & $\mathbf{2 5 2 , 9 2 8}$ & $\mathbf{2 2 2 , 1 2 3}$ & $\mathbf{1 2 0 , 6 1 0}$ & $\mathbf{2 0 1 , 0 9 4}$ \\
\hline Deuda a L. P. & 124,347 & 240,688 & 216,361 & 107,444 & 190,308 \\
\hline Otros pasivos & 9,957 & 12,240 & 5,762 & 13,166 & 10,786 \\
\hline TOTAL PASIVOS & $\mathbf{6 2 7 , 0 4 5}$ & $\mathbf{6 8 8 , 3 0 1}$ & $\mathbf{5 8 4 , 2 2 6}$ & $\mathbf{5 8 5 , 1 7 2}$ & $\mathbf{5 3 4 , 9 9 7}$ \\
\hline PATRIMONIO & $\mathbf{2 2 5 , 0 8 0}$ & $\mathbf{2 3 1 , 9 0 7}$ & $\mathbf{2 3 9 , 0 7 9}$ & $\mathbf{2 1 8 , 2 8 0}$ & $\mathbf{2 1 2 , 0 8 2}$ \\
\hline Capital social & 173,341 & 173,341 & 173,341 & 156,119 & 137,808 \\
\hline Capital adicional & 44,637 & 44,637 & 44,637 & 41,679 & 41,679 \\
\hline Reservas y otros & 11,587 & 12,674 & 31,941 & 9,021 & 7,712 \\
\hline $\begin{array}{l}\text { Resultados } \\
\text { acumulados }\end{array}$ & $(4,485)$ & 1,255 & $(10,840)$ & 11,461 & 24,883 \\
\hline $\begin{array}{l}\text { TOTAL PASIVOS Y } \\
\text { PATRIMONIO }\end{array}$ & $\mathbf{8 5 2 , 1 5 2}$ & $\mathbf{9 2 0 , 2 0 8}$ & $\mathbf{8 2 3 , 3 0 5}$ & $\mathbf{8 1 2 , 4 2 8}$ & $\mathbf{7 5 2 , 5 9 7}$ \\
\hline
\end{tabular}

Como se puede apreciar en el año 2001, la empresa mostraba de acuerdo a su balance general un activo total de 852 millones de soles con un patrimonio de 225 millones y un pasivo total de 627 millones. De esta manera, la estructura de capital que presentaba era de $26 \%$ de capital propio y $74 \%$ de recursos de terceros. En el año 2,000 la estructura era de $25 \%$ de capital propio y $75 \%$ de terceros. En 1,999 la estructura era de $29 \%$ de capital propio y $71 \%$ de capital de terceros. En 1,998 la estructura era de $27 \%$ de capital propio y $73 \%$ de capital de terceros. Y en 1,997 el capital propio financiaba el $28 \%$ y el $72 \%$ con recursos de terceros. 


\section{Raimundo Renaun Pacheco Mexzon}

A continuación se presenta el resumen del estado de ganancias y pérdidas para el periodo 1997-2001.

\section{Resumen histórico del estado de ganancias y pérdidas}

En miles de soles

\begin{tabular}{|l|c|c|c|c|c|}
\hline RUBROS & $\mathbf{2 0 0 1}$ & $\mathbf{2 0 0 0}$ & $\mathbf{1 9 9 9}$ & $\mathbf{1 9 9 8}$ & $\mathbf{1 9 9 7}$ \\
\hline Ventas netas & 608,711 & 577,672 & 676,775 & 604,245 & 539,811 \\
\hline Otros ingresos & 12,641 & 16,563 & 31,621 & & \\
\hline TOTAL INGRESOS & $\mathbf{6 2 1 , 3 5 2}$ & $\mathbf{5 9 4 , 2 3 5}$ & $\mathbf{7 0 8 3 9 6}$ & $\mathbf{6 0 4 , 2 4 5}$ & $\mathbf{5 3 9 , 8 1 1}$ \\
\hline Costo de ventas & 466,657 & 423,640 & 519,496 & 443,321 & 398,622 \\
\hline Utilidad bruta & $\mathbf{1 5 4 , 6 9 5}$ & $\mathbf{1 7 0 , 5 9 5}$ & $\mathbf{1 8 8 , 9 0 0}$ & $\mathbf{1 6 0 , 9 2 4}$ & $\mathbf{1 4 1 , 1 8 9}$ \\
\hline Gastos operativos & 128,610 & 125,701 & 142,481 & 141,573 & 117,326 \\
\hline Utilidad operativa & $\mathbf{2 6 , 0 8 5}$ & $\mathbf{4 4 , 8 9 4}$ & $\mathbf{4 6 , 4 1 9}$ & $\mathbf{1 9 , 3 5 1}$ & $\mathbf{2 3 , 8 6 1}$ \\
\hline $\begin{array}{l}\text { Otros ingresos y } \\
\text { egresos }\end{array}$ & $(32,673)$ & $(41,451)$ & $(57,406)$ & $(5,173)$ & 12,693 \\
\hline $\begin{array}{l}\text { Utilidad entes de } \\
\text { participaciones }\end{array}$ & $\mathbf{( 6 , 5 8 8 )}$ & $\mathbf{3 , 4 4 3}$ & $\mathbf{( 1 0 , 9 8 7 )}$ & $\mathbf{1 4 , 1 7 8}$ & $\mathbf{3 6 , 5 5 4}$ \\
\hline Participaciones & 252 & $(527)$ & 0 & $(1,240)$ & $(2,924)$ \\
\hline $\begin{array}{l}\text { Utilidad antes de } \\
\text { impuestos }\end{array}$ & $\mathbf{( 6 , 3 3 6 )}$ & $\mathbf{2 , 9 1 6}$ & $\mathbf{( 1 0 , 9 8 7 )}$ & $\mathbf{1 2 , 9 3 8}$ & $\mathbf{3 3 , 6 3 0}$ \\
\hline Impuesto a la renta & 596 & $(477)$ & 147 & $(203)$ & $(5,982)$ \\
\hline Utilidad neta & $\mathbf{( 5 , 7 4 0 )}$ & $\mathbf{2 , 4 3 9}$ & $\mathbf{( 1 0 , 8 4 0 )}$ & $\mathbf{1 2 , 7 3 5}$ & $\mathbf{2 7 , 6 4 8}$ \\
\hline
\end{tabular}

De acuerdo a los datos históricos, se aprecia que los ingresos por ventas han tenido un comportamiento cíclico llegando en el año 2001 a 621 millones 352 mil soles. La utilidad operativa alcanzó a 26 millones 85 mil soles y se llegó a una pérdida neta de 5 millones 740 mil soles.

Tomando como base la data histórica, se calculará el valor de la empresa, en dólares, considerando un escenario futuro de cinco años como periodo explícito. 


\section{Pensamiento Crítico Vol.I7. No I}

\section{Pronósticos y proyecciones}

\section{Ingresos por ventas}

Tomando en consideración que Ferreyros es una empresa proveedora de bienes de capital para los sectores económicos más importantes del país como son la minería, la agricultura, la pesca y el transporte, sus ventas están íntimamente ligadas al crecimiento de la economía del Perú. De acuerdo a las cifras macroeconómicas y a los estimaciones del crecimiento del país, que estiman un nivel de crecimiento entre el 4 y 6 por ciento anual, consideramos, en una posición optimista que el crecimiento de las ventas deben alcanzar por lo menos el $5 \%$ en los dos primeros años, el $4 \%$ en el tercer año y $3 \%$ en los dos últimos años. En este sentido, el crecimiento de las ventas tendrá el siguiente comportamiento:

\section{Proyecciones de las ventas}

\section{En miles de US\$}

\begin{tabular}{|l|c|c|c|c|c|c|}
\hline \multicolumn{1}{|c|}{ AÑOS } & $\mathbf{0}$ & $\mathbf{1}$ & $\mathbf{2}$ & $\mathbf{3}$ & $\mathbf{4}$ & $\mathbf{5}$ \\
\hline Ventas & 172,598 & 181,228 & 190,289 & 197,901 & 203,838 & 209,953 \\
\hline Crecimiento & & $5 \%$ & $5 \%$ & $4 \%$ & $3 \%$ & $3 \%$ \\
\hline
\end{tabular}

\section{Costo de ventas}

El costo de ventas que ha tenido un comportamiento histórico entre el $73 \%$ y $76 \%$ de las ventas, debe de llegar en el cuarto año al 70\%, que es el nivel considerado como eficiente en empresas de estas características. Por lo tanto, el costo de ventas tendrá el siguiente comportamiento:

\section{Proyecciones del costo de ventas}

\section{En miles de US\$}

\begin{tabular}{|l|c|c|c|c|c|}
\hline \multicolumn{1}{|c|}{ AÑOS } & $\mathbf{1}$ & $\mathbf{2}$ & $\mathbf{3}$ & $\mathbf{4}$ & $\mathbf{5}$ \\
\hline Costo de ventas & 130,484 & 135,105 & 140,509 & 142,686 & 146,967 \\
\hline $\begin{array}{l}\text { Porcentaje de las } \\
\text { ventas }\end{array}$ & $72 \%$ & $71 \%$ & $71 \%$ & $70 \%$ & $70 \%$ \\
\hline
\end{tabular}




\section{Raimundo Renaun Pacheco Mexzon}

\section{Gastos de venta y administrativos}

Los gastos operativos que han tenido un comportamiento entre $23 \%$ y $21 \%$ de las ventas, deben llegar como máximo a un $20 \%$ que es un nivel aceptable para este tipo de negocios. Por lo tanto, tendrán el siguiente comportamiento:

\section{Proyecciones de los gastos operativos \\ En miles de US\$}

\begin{tabular}{|l|c|c|c|c|c|}
\hline \multicolumn{1}{|c|}{ AÑN } & $\mathbf{1}$ & $\mathbf{2}$ & $\mathbf{3}$ & $\mathbf{4}$ & $\mathbf{5}$ \\
\hline Gastos operativos & 36,246 & 38,058 & 39,580 & 40,768 & 41,991 \\
\hline $\begin{array}{l}\text { Porcentaje del costo } \\
\text { de ventas }\end{array}$ & $20 \%$ & $20 \%$ & $20 \%$ & $20 \%$ & $20 \%$ \\
\hline
\end{tabular}

\section{Inversiones}

Para tener la infraestructura modernizada y lograr mantenerse como líder en el ramo, se estima una inversión de 4 millones 167 mil dólares en el tercer año básicamente para equipamiento de talleres de mantenimiento. Esta inversión se estima permitiría incrementar los ingresos en $1 \%$ en forma continua a partir del sexto año.

\section{Capital de trabajo}

El capital de trabajo que en el año 2001 fue negativo en 11 millones 86 mil dólares, debe comportarse acorde con las ventas, es decir $5 \%$ en los dos primeros años, $4 \%$ en el tercero, y $3 \%$ en los dos últimos años. Por lo tanto, el comportamiento del capital de trabajo será:

\section{Crecimiento del capital de trabajo}

\section{En miles de US\$}

\begin{tabular}{|c|c|c|c|c|c|}
\hline AÑOS & $\mathbf{1}$ & $\mathbf{2}$ & $\mathbf{3}$ & $\mathbf{4}$ & $\mathbf{5}$ \\
\hline $\begin{array}{c}\text { Incremento del } \\
\text { capital de trabajo }\end{array}$ & $(554)$ & $(582)$ & $(605)$ & $(623)$ & $(642)$ \\
\hline Porcentaje & $5 \%$ & $5 \%$ & $4 \%$ & $3 \%$ & $3 \%$ \\
\hline
\end{tabular}




\section{Depreciación}

La depreciación acumulada de un año fue de 53'577,000 soles y en el año anterior fue de 44'594,000, por lo tanto, la depreciación anual para los tres primeros años será de 8'983,000, equivalente a 2'495,000 dólares. Para los próximos dos años será de 3’329,000 incluyendo la depreciación de la nueva inversión.

\section{Cálculo de la depreciación}

\begin{tabular}{|ll|c|}
\hline \multicolumn{1}{|c|}{ RUBROS } & MILES \\
\hline Depreciación acumulada año & (Soles) & 53,577 \\
\hline Depreciación acumulada año anterior & (Soles) & 44,594 \\
\hline Diferencia & (Soles) & 8,983 \\
\hline Depreciación anual & US\$ & $\mathbf{2 , 4 9 5}$ \\
\hline Depreciación de nueva inversión en 5 años & US\$ & 833 \\
\hline Depreciación año 4 y 5 & US\$ & $\mathbf{3 , 3 2 9}$ \\
\hline
\end{tabular}

\section{Flujo de caja libre proyectado}

La valorización de la empresa se hará utilizando el método del flujo de caja descontado, por ser la empresa un negocio en marcha y líder en su rubro en el país.

Con la información presentada en el punto anterior, el flujo de caja libre generado por la empresa en los próximos cinco años correspondientes al periodo explícito será:

\section{Flujo de caja libre proyectado}

\section{En miles de US\$}

\begin{tabular}{|l|c|c|c|c|c|}
\hline \multicolumn{1}{|c|}{ AÑOS } & $\mathbf{1}$ & $\mathbf{2}$ & $\mathbf{3}$ & $\mathbf{4}$ & $\mathbf{5}$ \\
\hline Ventas & 181,228 & 190,289 & 197,901 & 203,838 & 209,953 \\
\hline Costo de ventas & 130,484 & 135,105 & 140,509 & 142,686 & 146,967 \\
\hline Gastos operativos & 36,246 & 38,058 & 39,580 & 40,768 & 41,991 \\
\hline $\begin{array}{l}\text { Utilidad antes de } \\
\text { intereses } e \text { impuestos }\end{array}$ & 14,498 & 17,126 & 17,811 & 20,384 & 20,995 \\
\hline
\end{tabular}


Raimundo Renaun Pacheco Mexzon

\begin{tabular}{|l|c|c|c|c|c|}
\hline $\begin{array}{l}\text { Utilidad antes de } \\
\text { intereses después } \\
\text { de Impuestos (tasa } \\
30 \% \text { ) }\end{array}$ & 10,149 & 11,988 & 12,468 & 14,269 & 14,697 \\
\hline (+) Depreciación & 2,495 & 2,495 & 2,495 & 3,329 & 3,329 \\
\hline $\begin{array}{l}\text { (-) Desembolso de } \\
\text { capital por inversión }\end{array}$ & 2,495 & 2,495 & 6,662 & 3,329 & 3,329 \\
\hline $\begin{array}{l}\text { (-) Incremento K. } \\
\text { Trabajo }\end{array}$ & $(554)$ & $(582)$ & $(605)$ & $(623)$ & $(642)$ \\
\hline Flujo Caja Libre & $\mathbf{1 0 , 7 0 3}$ & $\mathbf{1 2 , 5 7 0}$ & $\mathbf{8 , 9 0 6}$ & $\mathbf{1 4 , 8 9 2}$ & $\mathbf{1 5 , 3 3 9}$ \\
\hline
\end{tabular}

\section{Estructura de capital}

De acuerdo a la información presentada en el resumen histórico de los balances para el periodo 1997-2001 la estructura de capital de la empresa se ha modificado paulatinamente presentando para el año 2001 un $26 \%$ de patrimonio y un $74 \%$ de deuda. El comportamiento de la estructura de capital se muestra a continuación:

\section{Comportamiento de la estructura de capital}

\section{En miles de Soles}

\begin{tabular}{|l|c|c|c|c|c|}
\hline & $\mathbf{2 0 0 1}$ & $\mathbf{2 0 0 0}$ & $\mathbf{1 9 9 9}$ & $\mathbf{1 9 9 8}$ & $\mathbf{1 9 9 7}$ \\
\hline TOTAL ACTIVOS & $\mathbf{8 5 2 , 1 5 2}$ & $\mathbf{9 2 0 , 2 0 8}$ & $\mathbf{8 2 3 , 3 0 5}$ & $\mathbf{8 1 2 , 4 2 8}$ & $\mathbf{7 5 2 , 5 9 7}$ \\
\hline TOTAL PASIVOS & 627,072 & 688,301 & 584,226 & 585,172 & 534,997 \\
\hline & & & & & \\
\hline PATRIMONIO & 225,080 & 231,907 & 239,079 & 218,280 & 212,082 \\
\hline $\begin{array}{l}\text { Patrimonio entre } \\
\text { activo total }\end{array}$ & $\mathbf{2 6 \%}$ & $\mathbf{2 5 \%}$ & $\mathbf{2 9 \%}$ & $\mathbf{2 7 \%}$ & $\mathbf{2 8 \%}$ \\
\hline $\begin{array}{l}\text { Pasivos entre } \\
\text { activo total }\end{array}$ & $\mathbf{7 4 \%}$ & $\mathbf{7 5 \%}$ & $\mathbf{7 1 \%}$ & $\mathbf{7 3 \%}$ & $\mathbf{7 2 \%}$ \\
\hline TOTAL & $\mathbf{1 0 0 \%}$ & $\mathbf{1 0 0 \%}$ & $\mathbf{1 0 0 \%}$ & $\mathbf{1 0 0 \%}$ & $\mathbf{1 0 0 \%}$ \\
\hline
\end{tabular}




\section{Pensamiento Crítico Vol.17. No I}

\section{Cálculo del costo del capital propio}

Para calcular el costo del capital propio se suele utilizar las condiciones presentadas en el mercado considerándose los riesgos respectivos. Es a partir del modelo CAPM (Capital Assets Pricing Model) que se puede calcular una tasa de rendimiento esperado (Tresp) para el accionista, que estaría definiendo su costo de oportunidad. El modelo que se resume en la siguiente fórmula nos permitió calcular el costo del capital propio.

$$
\text { Tresp }=\mathrm{TLr}+\mathrm{B}(\mathrm{RPM}-\mathrm{TLr})
$$

TLr es la tasa libre de riesgo y se asumió que su valor en el mercado es de 5.53\% tomando en consideración el rendimiento que pagan los Bonos en el mercado americano.

(RPM - TLr) es la prima del mercado (PRM) y para el caso de empresas peruanas está relacionada con la diferencia entre lo que pagan los Bonos Brady y la tasa del Bono Americano. En este caso se asume su valor de 5.7\%. Además se debe tener en cuenta que en el Perú, para cualquier actividad, se podría utilizar este valor ya que en el mercado americano se considera como prima de riesgo que ha sido calculado como el promedio geométrico del índice S \& P 500.

"B" conocido como beta, es el factor que determina las condiciones de riesgo con las probabilidades de rendimientos esperados en el mercado a través de la relación de la covarianza y la varianza. En el mercado americano se estima para las empresas del sector de extracción de recursos naturales y sin apalancamiento un beta promedio de 1.7; sin embargo, para empresas líderes el beta oscila entre menor que cero y 1.2 dependiendo del riesgo. Como Ferreyros es una empresa líder en el Perú, el beta considerado es de 1.1 , este resulta de ajustar 1.7 a un nivel de riesgo manejable del $64.5 \%(1.7 * 0.645=$ $1.1)$.

En consecuencia, el costo del capital propio para Ferryros se estimó de la siguiente manera:

$$
\mathrm{Ck}_{\mathrm{p}}=\mathrm{TLr}+\mathrm{B}(\mathrm{RPM}-\mathrm{TLr})
$$


$\mathrm{Ck}_{\mathrm{p}}=5.53+1.1 *(11.23-5.53)$

$$
\mathrm{Ck}_{\mathrm{p}}=11.80 \%
$$

\section{Cálculo del costo de la deuda}

Para calcular el costo de la deuda se han tomado los datos históricos de los estados financieros. Se determinó una tasa en soles de $12 \%$ anual. Esta tasa ajustada a la tasa equivalente en dólares, resultó una tasa equivalente de 4.67\%. En consecuencia, la tasa para la deuda se estimo en $5 \%$ anual.

Cálculo del costo de la deuda

\begin{tabular}{|l|c|}
\hline \multicolumn{1}{|c|}{ RUBROS } & MILES DE SOLES \\
\hline \multicolumn{1}{|c|}{ Gastos financieros } & $\mathbf{5 6 , 7 3 2}$ \\
\hline - Sobregiros y préstamos bancarios & 146,871 \\
\hline - Vencimiento corriente, deuda largo plazo & 199,806 \\
\hline - Deuda a largo plazo & 124374 \\
\hline Total deuda & $\mathbf{4 7 1 , 0 5 1}$ \\
\hline Gastos financieros entre deuda & $\mathbf{1 2 . 0 4 \%}$ \\
\hline Costo promedio deuda en US\$ & $7 \%$ \\
\hline Tasa equivalente en US\$ & $\mathbf{( 1 + 0 . 1 2 )} /(\mathbf{1 + 0 . 0 7 )}=\mathbf{4 . 6 7 \%}=\mathbf{5 \%}$ \\
\hline
\end{tabular}

\section{Cálculo del costo de capital promedio ponderado (wacc)}

Para traer a valor presente el flujo de caja libre debemos calcular previamente el costo promedio ponderado de capital (WACC) utilizando la siguiente fórmula:

$$
\mathrm{WACC}=\mathrm{P} /(\mathrm{P}+\mathrm{D}) *(\mathrm{~T} \operatorname{lr}+\mathrm{B} * \mathrm{PRM})+\mathrm{D} /(\mathrm{P}+\mathrm{D}) * \mathrm{kd}(1-\mathrm{T})
$$


De acuerdo a la información obtenida, los valores para calcular el WACC son:

$\begin{array}{ll}\mathrm{P} /(\mathrm{P}+\mathrm{D}) & =26 \% \\ \mathrm{D} /(\mathrm{P}+\mathrm{D}) & =74 \% \\ \mathrm{Tl} & =5.53 \% \\ \mathrm{~B} & =1.1 \\ \mathrm{PRM} & =5.70 \% \\ \mathrm{Kd} & =5.00 \% \\ \mathrm{~T} & =30 \%\end{array}$

Al reemplazar los datos en la fórmula se obtiene un WACC de 5.66\%.

$$
\mathrm{WACC}=5.66 \%
$$

Valor Actual del flujo de caja libre del periodo explícito (VAPE)

\begin{tabular}{|c|c|c|c|}
\hline AÑOS & FLUJO & $\begin{array}{c}\text { FACTOR } \\
\mathbf{5 . 6 6 \%}\end{array}$ & V A \\
\hline 1 & 10,703 & 0.94645 & 10,130 \\
\hline 2 & 12,570 & 0.89577 & 11,260 \\
\hline 3 & 8,906 & 0.84780 & 7,551 \\
\hline 4 & 14,892 & 0.80240 & 11,949 \\
\hline 5 & 15,339 & 0.75943 & 11,649 \\
\hline VAPE & & & $\mathbf{5 2 , 5 3 9}$ \\
\hline
\end{tabular}

\section{Cálculo del valor continuo}

Como la empresa es un negocio en marcha, se estima que a partir del sexto año habrá un flujo continuo con un crecimiento de las ventas en $1 \%$ por lo tanto, para el año sexto el flujo de caja libre para el periodo continuo será de $15,339 * 1.01=15,492$. Así mismo, el retorno sobre la nueva inversión será de $1 \%$ en forma continua 


\section{Raimundo Renaun Pacheco Mexzon}

Con esta información se calcula el valor continuo que será igual a:

$$
\begin{aligned}
& \mathrm{VC}=\mathrm{FC} \text { Libre año } 6 /(\text { WACC }- \text { retorno nueva inversión) } \\
& \mathrm{VC}=15,492 /(5.66 \%-1 \%) \\
& \mathrm{VC}=532,594
\end{aligned}
$$

\section{Valor actual del valor continuo}

Como el costo de capital promedio ponderado (WACC) es $5.66 \%$ el valor presente o valor actual de este flujo para el periodo continuo sería:

$$
\begin{aligned}
& \mathrm{VAPC}=532,492 /(1+0.0566) \wedge 6 \\
& \mathrm{VAPC}=239,594
\end{aligned}
$$

\section{Valor de la empresa en el mercado}

El valor de la empresa será:

$$
\begin{aligned}
& \mathrm{VE}=\mathrm{VAPE}+\mathrm{VAPC} \\
& \mathrm{VE}=52,539+239,056 \\
& \mathrm{VE}=\mathrm{US} \$ 291^{\prime} 595,000
\end{aligned}
$$

De acuerdo a los resultados obtenidos podemos decir que la empresa, pese a tener pérdidas en algunos años históricos, tiene un valor en el mercado de 291 millones 595 mil dólares, cifra que comparada con los activos totales que en el balance histórico llegan a 236 millones 709 mil dólares, su venta deja un excedente de 54 millones 887 mil dólares a favor de los actuales accionistas.

\section{Conclusiones}

1. De acuerdo a los supuestos utilizados y a las condiciones históricas que presenta la empresa, al utilizar el método de valorización basado en el Flujo de Caja Libre Descontado, se aprecia que el valor de la empresa supera el valor en libros, beneficiando su venta a los actuales dueños. 


\section{Pensamiento Crítico Vol.I7. No I}

2. Utilizando el "Flujo de Caja Libre" y determinando una adecuada estructura de capital se maximiza el valor de la empresa como se demuestra en el caso práctico.

3. Esta metodología nos permite maximizar el valor de la empresa en el mercado considerando que es un negocio en marcha y al venderla o fusionarse con otra empresa se consiguen mejores resultados para los accionistas.

\section{RECOMENDACIONES}

1. Para que esta situación se cumpla es recomendable que la empresa haga una reingeniería de costos para lograr un nivel competitivo del costo de ventas y los gastos operativos.

2. Si bien es cierto, la empresa muestra un valor aceptable, debe plantearse un plan financiero a largo plazo que le permita disminuir paulatinamente el alto nivel de endeudamiento que tiene. Hay que tener presente que un alto endeudamiento en condiciones no muy favorables del mercado incrementan el riesgo financiero. Una empresa muy apalancada cuando las ventas se quedan estacionarias o caen, caen también sus rendimientos del patrimonio ( $\mathrm{ROE}$ ).

\section{Bibliografía}

ALIAGA L. Rafael y SOUSA DE BARBIERI Lorenzo, Banca de inversión; Edit. Publicaciones Universidad de Piura, 1986.

BELLIDO S. Pedro, Administración financiera, Edit. Técnico Científica, 1989. Capítulo 17: Págs 245 272, Capítulo 18: Págs 277 - 297.

BOLTEN Esteven Manual de administración financiera, Edit. Ciencia y técnica. Limusa 1987., Capítulo 8: Págs. 351 - 378, Capítulo 10: Págs. 423 - 457

DIÉZ DE CASTRO, Luís y MASCARREÑAS, Juan, Ingeniería financiera: la gestión en los mercados financieros internacionales, Edit. McGRAW-HILL, 1998. Págs 91-92. 


\section{Raimundo Renaun Pacheco Mexzon}

FERNÁNDEZ Pablo Valoración de empresas Edit. Gestión 2000 S.A. 1999. Capítulos 1, 14, 15, 16, 19 y 20.

PACHECO M., Raimundo "ADRs y el Valor de La Empresa" Tesis para optar el Grado Académico de Magister en Economía mención Finanzas, Pags 11-13 y 47-50

PACHECO M., Raimundo "free cash flow y el valor de la empresa", Artículo en la revista Pensamiento Crítico N. ${ }^{\circ} 16$ de IIE.

VAN HORNE, James, Administración financiera Edit. Contabilidad Moderna Buenos Aires, 1973. Capítulo VII: Págs. 260 - 286, Capítulo VIII: Págs. 300 - 313.

http://www.pla.net.py/cnv/orientando, Glosario

http://www.superval.gob.sv/html, Glosario

http://www.corfinsura.com/español, Glosario 